\title{
INVESTIGACIONES
}

\section{Análisis descriptivo del juego como herramienta para aprender sobre el patrimonio cultural: estudio de caso}

\author{
Descriptive analysis of the game as a tool to learn about cultural heritage: \\ case study
}

\begin{abstract}
Análise descritiva do jogo como ferramenta de aprendizagem do patrimônio cultural: estudo de caso
\end{abstract}

\author{
Mariela Monserrath Barbecho Benalcázara , Jorge Luis Uyaguari Guamán ${ }^{a}$, \\ Ángel Torres-Toukoumidis ${ }^{a}$ \\ ${ }^{a}$ Universidad Politécnica Salesiana. Ecuador. \\ mbarbechob1@est.ups.edu.ec, juyaguarig@est.ups.edu.ec, atorrest@ups.edu.ec
}

\begin{abstract}
RESUMEN
Estudios previos han mostrado que el juego es uno de los medios de transmisión de costumbres que se manifiesta en todos los seres humanos. Por ende, los salones de clase podrían ser espacios lúdicos de transmisión de saberes y cultura. De allí que este análisis tiene como propósito conocer la influencia de las metodologías lúdicas en el aprendizaje de la cultura en niños y adolescentes de dos establecimientos educativos, determinando cómo la enseñanza favorece al desarrollo cultural. La investigación se basa en un enfoque cuantitativo de hipótesis descriptiva, con una muestra de 80 estudiantes de dos Instituciones Educativas de la ciudad de Cuenca-Ecuador, para lo cual se aplicó una encuesta pre-test y pos-test la actividad lúdica enfocada en la cultura. Los resultados señalaron avances significativos en cuanto al interés demostrado por niños y adolescentes respecto al uso de juegos para la enseñanza de la cultura. Por consiguiente, se concluye que las metodologías lúdicas favorecen al aprendizaje cultural en los salones de clase, en tanto, debe insistirse su incorporación como didáctica formativa en las aulas.
\end{abstract}

Palabras claves: Cultura, juego, educación, tradiciones, metodologías de la enseñanza, interacciones sociales, aprendizaje activo.

\begin{abstract}
Previous studies have shown that play is one of the means of transmitting customs that are manifested in all human beings. Therefore, classrooms could be playful spaces for the transmission of knowledge and culture. Hence, this analysis aims to understand the influence of playful methodologies in the learning of culture in children and adolescents in two educational establishments, determining how teaching favors cultural development. The research is based on a quantitative approach of a descriptive hypothesis, with a sample of 80 students from two educational institutions in the city of Cuenca-Ecuador, for which a pre-test and post-test survey was applied to the ludic activity focused on culture. The results showed significant progress in the interest shown by children and adolescents in the use of games for teaching culture. Therefore, it is concluded that playful methodologies favor cultural learning in the classrooms, while their incorporation as formative didactics in the classroom.
\end{abstract}

Key words: Culture, play, education, traditions, teaching methodologies, social interactions, active learning. 


\section{RESUMO}

Estudos anteriores demonstraram que o jogo é um dos meios de transmissão de costumes que se manifesta em todos os seres humanos. Portanto, as salas de aula podem ser espaços lúdicos de transmissão de conhecimento e cultura. Assim, o objetivo desta análise é conhecer a influência das metodologias lúdicas na aprendizagem da cultura em crianças e adolescentes de dois estabelecimentos de ensino, determinando como o ensino favorece o desenvolvimento cultural. A pesquisa baseia-se em uma abordagem quantitativa de hipótese descritiva, com uma amostra de 80 alunos de duas Instituições de Ensino da cidade de Cuenca-Equador, para as quais foi aplicado um pré-teste e um pós-teste survey à atividade lúdica focada no cultura. Os resultados indicaram avanços significativos quanto ao interesse de crianças e adolescentes quanto ao uso de jogos para o ensino da cultura. Conseqüentemente, conclui-se que as metodologias lúdicas favorecem a aprendizagem cultural em sala de aula, devendo ser insistida em sua incorporação como didática formativa em sala de aula.

Palavras-chave: Cultura, brincar, educação, tradições, metodologias de ensino, interações sociais, aprendizagem ativa.

\section{INTRODUCCIÓN}

Uno de los retos que deben enfrentarse los profesionales de la educación es, la atención a la diversidad cultural en los salones de clase. La pluralidad cultural es una característica de nuestra sociedad, vivimos en un entorno donde las tradiciones distintas y los colectivos humanos que las practican habitan en un mismo entorno social (Mestres, 2009). De hecho, Porro (2014) admite que, en esta sociedad globalizada, se da mayor preferencia a productos extranjeros, se imita otras culturas. Esto se debe principalmente a que existe un desconocimiento de la propia cultura que tiene como consecuencia la pérdida de la capacidad de actuar como fuente de identidad y organización colectiva, por tal razón se marca una transformación en el ámbito del consumo cultural, que pone en evidencia que las culturas originarias junto con sus costumbres y tradiciones han sido sustituidas por una mezcla de aficiones que manifiestan los individuos en sus prácticas y consumos culturales.

Por ende, es importante desarrollar la identidad nacional en los establecimientos educativos en donde se puede trabajar el patrimonio cultural a la par con la malla curricular, como consecuencia se estaría generando un medio esencial para fortalecer nuestra propia identidad y a la vez aprender más sobre temas culturales de una manera más activa. Según la ley Orgánica de Educación Intercultural Bilingüe que entró en vigor el 31 de marzo del 2011 en Ecuador, el artículo 2. Principios, literal z) Interculturalidad y plurinacionalidad expresa:

La interculturalidad y plurinacionalidad garantizan a los actores del Sistema el conocimiento, el reconocimiento, el respeto, la valoración, la recreación de las diferentes nacionalidades, culturas y pueblos que conforman el Ecuador y el mundo; así como sus saberes ancestrales, propugnando la unidad en la diversidad, propiciando el diálogo intercultural e intracultural, y propendiendo a la valoración de las formas y usos de las diferentes culturas que sean consonantes con los derechos humanos (Constitución política del Ecuador, 2011, Artículo 2).

En otras palabras, el ámbito educativo tiene como responsabilidad el velar por que sus actores convivan en una sociedad cultural; por lo es importante trabajar desde las aulas de clases en temas de cultura y patrimonio. Con el fin de que esto suceda se requiere de una metodología que incite de forma activa al aprendizaje y transferencia de la cultura y 
patrimonio. De hecho, Aizencang (2005) ha considerado al juego como uno de los medios más influyentes para la transmisión de costumbres, especialmente, en la etapa infantil y adolescente. Por lo que en el campo educativo este tipo de estrategias pueden jugar un papel importante para que las tradiciones y conocimientos culturales se transmitan en los salones de clase.

En consecuencia, este artículo se fundamentará en la necesidad de revalorizar la cultura en el ámbito educativo mediante un proceso investigativo sobre la influencia de las metodologías lúdicas en el aprendizaje cultural y patrimonial de manera significativa y funcional, remarcando al juego como una herramienta básica en la educación, y por ende, buscando sentenciar que las aulas de clase de las escuelas de Básica podrían ser espacios lúdicos propicios para la transmisión de saberes y cultura de un pueblo.

\section{MARCO TEÓRICO}

\subsection{LA CULTURA Y SU ENSEÑANZA}

La mente no podría existir si no fuera por la cultura, por ende, la educación no debe visualizarse de manera aislada de la sociedad; sino entender que ambos son parte de un todo cultural, tanto el aprendizaje como el conocimiento están interrelacionados en un contexto cultural (Bruner, 2015). En otras palabras, la cultura se aprende y se transmite, por lo que su aprendizaje sugiere de un proceso de educación que se viene dando a lo largo de la vida de un ser humano. De tal modo, Ferro (2013) asevera que "El bagaje de los bienes culturales debe ser transmitido de una generación a otra... Es esta la función más general de la educación" (p. 15). Dicho de otra forma, la cultura no solo se aprende sino se enseña. Entonces es indudable que la escuela prepare al ser humano en aspectos técnicos, científicos y espirituales, siendo este último afectado en cierta manera por el mundo globalizado; perdiendo así poco a poco la cultura y la identidad nacional (Borroto, 2017). Es así como se le debe considerar al salón de clase como un parque de intercambio de experiencias, en donde se potencia las habilidades, capacidades, destrezas y actitudes adquiridas en su entorno social. En consecuencia, la escuela brinda un empoderamiento social y cultural (Martínez, 2008).

\subsection{EL JUEGO Y SUS DIFERENTES ASPECTOS}

Megías y Lozano (2019) mencionan que "el juego es una actividad lúdica que satisface necesidades innatas, implica participación activa y favorece su desarrollo integral en las dimensiones físico-sensorial, cognitiva y socio afectivas" (p. 9). De allí, que el juego se origina con el propio desarrollo de la sociedad y es una actividad muy significativa en la vida de las personas, porque conduce al hombre hacia la integración de un grupo social. Al mismo tiempo permite el intercambio y la modificación de las costumbres que caracterizan a cada colectividad. Además, la actividad lúdica no se puede heredar, esta transmisión se da por una herencia social, propiciando que las diferentes generaciones sean partícipes de actividades lúdicas a través del tiempo (Marcaida y Carreño, 2013).

El juego se ha vuelto un medio de sociabilización, un niño o adolescente cuando juega establece lazos de amistad, y a la vez resuelve problemas. Siendo la escuela un lugar para 
la transferencia cultural, y lo lúdico un medio para que se de este legado. Aseverando tal enunciado Marín (2018) expone que "el juego como metodología y estrategia, esencia de lo lúdico, puede transformar de forma radical y disruptiva el proceso de la enseñanza y del aprendizaje." (p. 10). Por consiguiente, con el aspecto lúdico se aprende afrontar desafíos, a seguir reglas, a establecer compromisos, a desarrollar nuestra creatividad y fantasía, poco a poco produciendo la integración social. Razón por la cual, al momento de jugar sin darse cuenta se tiende a dar más relevancia al proceso que al mismo resultado, en ese sentido se propende a un aprendizaje social (Loos y Metref, 2007).

Por otra parte, también tenemos que mencionar los juegos multiculturales que hace referencia a una gama de culturas que están enlazadas mediante lo lúdico aportando al fortalecimiento de la cultura (Capretti, 2011). Por esto, los juegos multiculturales abarcan también los juegos tradicionales que se han venido manteniendo de generación en generación (Bantulá y Mora, 2016). De este modo, los juegos tradicionales son los más apropiados para desarrollar un aprendizaje activo; ya que no son muy elaborados y sus reglas se pueden definir de acuerdo con el contexto o tema, por lo que son muy útiles para enseñar y aprender cultura (Cañizares y Carbonero, 2016).

\subsection{RELACIÓN ENTRE LA CULTURA, EDUCACIÓN Y JUEGO}

La relación existente entre cultura y educación es el hecho de que las interacciones sociales y el ambiente sociocultural son vitales para el desarrollo de la mente humana (Castorina y Dubrovsky, 2004). Considerando lo anteriormente mencionado, el ser humano no es un ente aislado que aprende de forma individual conocimientos, destrezas y actitudes, sino que el entorno cultural y social juega también un papel importante en su formación.

Es muy relevante reconocer cuál es la relación que existe en el juego, la educación y la cultura. Vial (1988) afirma: "el juego y la educación han marcado la Humanidad desde el momento en que ésta escapaba de los rigurosos determinismos de la supervivencia y de la animalidad" (p. 13). Es decir, los juegos como fenómeno cultural garantizan un fortalecimiento de los rasgos culturales de una nación, país, pueblo, comunidad e individuo (Paredes, 2003).

En definitiva, las actividades lúdicas, deportivas-recreativas o festivas han sido una constante humana; aparecen reflejadas en sus primeras manifestaciones culturales (Peñalva, Aguaded y Torres-Toukoumidis, 2019). Hacer arte o deporte es como jugar con las reglas, las leyes y la disciplina de una forma lúdica que implica goce individual y colectivo, experiencias extraordinarias, protagonismos, osadías, atrevimientos interesantes, sociabilidad, el probar las posibilidades de dar y recibir con esfuerzo y la increíble sensación de que estimen lo que hacen dentro de la familia, amigos, conocidos y sin embargo es insuficiente el reconocimiento de los procedimientos lúdicos en la dirección de las relaciones colectivas y personales de los educandos en el proceso educativo. A través de ellos las actividades resultan más interesantes, asequibles y atrayentes, se superan las dificultades y se crean condiciones más favorables para desarrollar la independencia, el interés por lo que les rodea y el espíritu de observación (Bermúdez, Díaz y Fernández, 2018, p. 175). 


\section{HIPÓTESIS DE LA INVESTIGACIÓN}

En base a las referencias teóricas citadas anteriormente, la hipótesis de la investigación es las metodologías lúdicas potencian el aprendizaje de la cultura y patrimonio en los salones de clase y fortalece el conocimiento sociocultural de niños y adolescentes.

\section{OBJETIVOS}

\subsection{OBJETIVO GENERAL}

Analizar la influencia de las metodologías lúdicas en el aprendizaje de la cultura del Ecuador en niños y adolescentes de dos instituciones educativas.

\subsection{OBJETIVOS ESPECÍFICOS}

- Identificar el impacto del uso de juegos en el aprendizaje de la cultura ancestral en estudiantes del nivel medio y superior.

- Determinar el efecto diferenciador que tienen las metodologías lúdicas en el aprendizaje de la cultura ancestral.

\section{METODOLOGÍA}

El diseño de investigación empleado fue cuasi experimental, de enfoque cuantitativo y alcance descriptivo, pues, muestra con precisión los ángulos y dimensiones de un fenómeno. Se asumió el estudio cuasi experimental debido a que no se estableció un grupo de control, sino que se aplicó la metodología lúdica a la muestra total obteniendo así una trazabilidad a dos grupos de niveles educativos e instituciones diferentes. Complementariamente, Hernández-Sampieri, Fernández y Baptista (2010) consideran el enfoque cuantitativo de alcance descriptivo logran especificar las características y propiedades con la finalidad de determinar tendencias prefiguradas de ciertos atributos dentro de un contexto particular.

\subsection{MUESTRA}

La muestra seleccionada fue no aleatoria abarcando a estudiantes de dos instituciones educativas de la ciudad de Cuenca: Ricardo Muñoz Chávez y Ulises Chacón, cuyos aprendizajes culturales están en formación. Se debe manifestar que los alumnos del uno y otro centro educativo desarrollaron las mismas encuestas y el mismo juego. Por un lado, la unidad educativa Ricardo Muñoz Chávez participó con cuarenta niños de edades entre 9 a 11 años, y, por otro lado, la escuela de educación básica Ulises Chacón, con cuarenta adolescentes, de edades entre 14 a 15 años. Cabe señalar que se considera niños o niñas a las personas comprendidas entre los 3 y 11 años; mientras que la adolescencia está determinada entre los 12 a 20 años (Papalia y Martorell, 2017). 


\subsection{METODOLOGÍA DEL JUEGO}

En el estudio se empleó un juego basado en el Twister, el mismo que fue modificado con preguntas de cultura ecuatoriana constituidas de acuerdo con la edad cronológica y al nivel de preparación, en primer lugar, se eligió un árbitro y una persona encargada de leer las preguntas para realizar los diferentes desafíos. Luego, se solicitó que los participantes coloquen un pie en cada círculo de colores para iniciar la actividad, inmediatamente el árbitro gira la ruleta y antes de que el participante realice el reto, la persona encargada lee en voz alta la pregunta, si acierta el participante sigue jugando, caso contario es remplazado por otro estudiante.

\subsection{RECOLECCIÓN DE DATOS}

El instrumento empleado fue la encuesta de tipo descriptiva; debido a que permite recolectar información de una forma estandarizada, mediante un cuestionario. El mismo estaba conformado por 10 ítems basados en dos escalas de sondeo: la de Likert, con un nivel de medición del 1 al 3; y la de tipo nominal. Dicho instrumento se organizó con base en dos criterios: aprendizaje de la cultura en el aula, 5 preguntas, y el juego en el aprendizaje de la cultura que contó también con 5 preguntas. El objetivo de este instrumento fue el de conocer las opiniones de los encuestados, además se utilizó emoticones para establecer las escalas de las preguntas.

Por otra parte, los ítems fueron de tipo cerradas y politómicas, el instrumento se aplicó en dos instancias; un pretest, al inicio de la investigación aplicado para determinar la percepción de los conocimientos previos sobre cultura, educación y juego; y un postest, luego de la aplicación del juego en el aula, puesto que se busca conocer cómo el juego intervino en el aprendizaje de la cultura. Por lo que las pruebas eran similares tanto en contenido como en número.

\subsection{VALIDACIÓN DEL INSTRUMENTO}

Inmediatamente del proceso de conformación del instrumento, se procedió a realizar una validación del contenido a través de cinco expertos del área de educación, definiendo el perfil de expertos como personas que poseen más de 20 años laborando en el ámbito de la pedagogía. A partir de los comentarios expuestos por los expertos, se resaltó que se trata de una encuesta adecuada y sencilla para el perfil de los destinarios; además, posee un buen carácter visual y didáctico. Por el contrario, se ejecutaron ajustes en el orden de respuestas de los ítems tres y seis; en la redacción de las preguntas ocho y nueve; y por último en el enunciado de la pregunta siete. Al mismo tiempo se efectúo una muestra piloto del $10 \%$ de los encuestados, obteniendo un 0,8 de Alfa de Cronbach determinando la fiabilidad de la herramienta.

\subsection{TIEMPO DE APLICACIÓN DEL INSTRUMENTO}

Esta investigación fue desarrollada aproximadamente en dos meses, es así, que el pretest se aplicó desde el viernes 13 hasta el martes 17 de septiembre del 2019; mientras que la actividad lúdica como metodología se ejecutó desde el 21 al 25 de octubre del 2019. 
Por último, el postest se planteó del 5 al 8 de noviembre. Los datos obtenidos fueron organizados a través del software SPSS versión 26.0 generando los siguientes hallazgos.

\section{RESULTADOS}

\subsection{RESULTADOS DEL PRETEST}

En el primer ítem, el 85\% de niños encuestados piensa que el aprendizaje de la cultura es importante; mientras que el $75 \%$ de adolescentes manifiesta de la misma manera. Sin embargo, se puede visualizar en el segundo ítem que; en ambos niveles de formación no existe la aplicación de metodologías lúdicas para la enseñanza de la cultural; ya que el 50\% de los niños y el 55\% de adolescentes responden que en sus centros educativos no existen formas dinámicas para este aprendizaje. Por consiguiente, el grado de conocimiento de cultura de niños y adolescentes es poco, el $77,50 \%$ en el primero y el $75 \%$ en el segundo.

Además, la forma cómo adquieren estos conocimientos es muy tradicional, poco atractiva y lúdica; el $45 \%$ y el $75 \%$ de adolescentes afirman que aprenden mediante libros y cuadernos, solamente el 10\% de niños y el 5\% de adolescentes expresan haber tenido un aprendizaje lúdico. No obstante, el $65 \%$ de niños y el $70 \%$ de adolescentes contestaron que el juego es un factor muy importante que ayuda a transmitir tradiciones y costumbres de un pueblo. En el sexto ítem se pudo conocer que el área de estudio con el que más han aprendido jugando los niños es la matemática con el 37,5\%, mientras que en los adolescentes es la ciencia con el 35\%. Por otro lado, solamente el 7,5\% de niños y el 2,5 de adolescentes enuncian que el juego al momento de aprender tradiciones y cultura de un pueblo es una pérdida de tiempo. De manera que la actividad más sobresaliente para aprender sobre la cultura de un pueblo fue la Danza con un $40 \%$ en niños y un $60 \%$ en los adolescentes. Por lo que al contestar sobre si es importante mezclar el aprendizaje de la cultura con el juego el $75 \%$ de niños y el $65 \%$ de adolescentes denotaron que a veces. Por último, el $90 \%$ de niños y el $45 \%$ de adolescentes formularon que les gustaría aprender la cultura jugando.

Tabla 1. Resultados del Pretest

\begin{tabular}{|l|l|c|c|}
\hline \multirow{2}{*}{ Ítem } & \multirow{2}{*}{ Respuesta } & \multicolumn{2}{c|}{$\%$} \\
\cline { 3 - 4 } & Sí & Niños & Adolescentes \\
\hline \multirow{2}{*}{$\begin{array}{l}\text { 1) ¿Crees que es importante la enseñanza } \\
\text { de cultura en el aula de clase? }\end{array}$} & No & 85 & 75 \\
\cline { 2 - 4 } & Tal vez & 0 & 5 \\
\hline \multirow{2}{*}{$\begin{array}{l}\text { 2) ¿En tu Institución Educativa se han } \\
\text { buscado formas dinámicas para enseñar } \\
\text { cultura? }\end{array}$} & Sí & 32,50 & 10 \\
\cline { 2 - 4 } & No & 50,00 & 55 \\
\cline { 2 - 4 } & Tal vez & 17,50 & 35 \\
\hline
\end{tabular}




\begin{tabular}{|c|c|c|c|}
\hline \multirow{3}{*}{$\begin{array}{l}\text { 3) ¿Cómo generalmente adquieres tus } \\
\text { conocimientos? }\end{array}$} & Nada & 0 & 0 \\
\hline & Poco & 77,5 & 75 \\
\hline & Mucho & 22,5 & 25 \\
\hline \multirow{5}{*}{$\begin{array}{l}\text { 4) ¿Cómo generalmente adquieres tus } \\
\text { conocimientos? }\end{array}$} & Memoria & 7,5 & 5 \\
\hline & Juegos & 10 & 5 \\
\hline & Videos & 35 & 15 \\
\hline & Libros/cuadernos & 45 & 75 \\
\hline & Otros & 2,5 & 0 \\
\hline \multirow{3}{*}{$\begin{array}{l}\text { 5) A través del juego se puede transmitir } \\
\text { costumbres y tradiciones de un pueblo. }\end{array}$} & Sí & 65 & 70 \\
\hline & No & 7,5 & 5 \\
\hline & Tal vez & 27,5 & 25 \\
\hline \multirow{5}{*}{$\begin{array}{l}\text { 6) ¿Con qué área del conocimiento has } \\
\text { aprendido jugando? }\end{array}$} & Cultura & 27,5 & 35 \\
\hline & Ciencia & 20 & 25 \\
\hline & Matemática & 37,5 & 15 \\
\hline & Lenguaje & 10 & 10 \\
\hline & Otros & 5 & 15 \\
\hline \multirow{5}{*}{$\begin{array}{l}\text { 7) ¿Qué le aporta el juego al momento de } \\
\text { aprender tradiciones y cultura de un } \\
\text { pueblo? }\end{array}$} & Inseguridad & 3,75 & 0 \\
\hline & $\begin{array}{l}\text { Transmite costumbres y } \\
\text { tradiciones }\end{array}$ & 40 & 100 \\
\hline & Es una pérdida de tiempo & 7,5 & 5 \\
\hline & $\begin{array}{l}\text { Desarrolla la comunicación } \\
\text { entre los compañeros/as y } \\
\text { docentes }\end{array}$ & 43,75 & 80 \\
\hline & No he jugado & 5 & 15 \\
\hline \multirow{5}{*}{$\begin{array}{l}\text { 8) ¿Qué actividad has puesto en práctica } \\
\text { para aprender sobre la cultura de un } \\
\text { pueblo? }\end{array}$} & Danza & 40 & 60 \\
\hline & Dramatización & 35 & 20 \\
\hline & Juegos & 10 & 15 \\
\hline & Pintura & 2,5 & 5 \\
\hline & Otros & 12,5 & 0 \\
\hline \multirow{3}{*}{$\begin{array}{l}\text { 9) ¿Es importante mezclar el aprendizaje } \\
\text { de la cultura con el juego? }\end{array}$} & Nunca & 0 & 5 \\
\hline & A veces & 75 & 65 \\
\hline & Siempre & 25 & 30 \\
\hline \multirow{3}{*}{$\begin{array}{l}\text { 10) ¿Te gustaría aprender la cultura } \\
\text { jugando? }\end{array}$} & Sí & 90 & 50 \\
\hline & No & 0 & 5 \\
\hline & Tal vez & 10 & 45 \\
\hline
\end{tabular}

La encuesta fue aplicada a niños y adolescentes de edades entre 9 a 15 años, antes de aplicar la metodología lúdica. Fuente: Elaboración propia. 


\subsection{RESULTADOS DEL POSTEST}

El 85\% de niños y adolescentes afirman que es transcendental el aprendizaje de la cultura. Asimismo, el $87,50 \%$ y el $75 \%$ de adolescentes piensan que las dinámicas y juegos realizados les ayudaron a crear un aprendizaje cultural. Y por último el $50 \%$ de estudiantes, tanto niños como adolescentes alegan que el aprendizaje sobre temas culturales, luego de realizarse el juego fue mucho. Por otra parte, el $50 \%$ y el $70 \%$ de adolescentes prefieren aprender cultura mediante el juego. Sin embargo, se puede visualizar que existe un porcentaje considerable respecto a la preferencia por aprender mediante videos, de tal manera que se puede observar un $42,50 \%$ en niños y $25 \%$ en adolescentes. No obstante, el $57,50 \%$ y el $70 \%$ de niños y adolescentes respectivamente manifestaron que a través del juego desarrollado pudo conocer costumbres y tradiciones de un pueblo. Dicho esto, se pudo conocer que los juegos realizados tuvieron una buena aceptación en los dos grupos de muestra; siendo así que el $75 \%$ de niños y el $90 \%$ de adolescentes opinaron que fue muy educativo y divertido. Al mismo tiempo opinaron el $41,25 \%$ de niños y el $50 \%$ de adolescentes que la actividad lúdica propició una comunicación asertiva entre los participantes. Además, $47 \%$ de niños y el $45 \%$ de adolescentes contestaron que el juego al momento de realizarlo brindó el conocimiento de costumbres y tradiciones del entorno.

Tabla 2. Resultados del Postest

\begin{tabular}{|c|c|c|c|}
\hline \multirow{2}{*}{ Ítem } & \multirow{2}{*}{ Respuesta } & \multicolumn{2}{|r|}{$\%$} \\
\hline & & Niños & Adolescentes \\
\hline \multirow{3}{*}{$\begin{array}{l}1 \text { ¿Consideras que es importante } \\
\text { aprender cultura en tu Institución } \\
\text { Educativa? }\end{array}$} & Sí & 85 & 85 \\
\hline & No & 0 & 0 \\
\hline & Tal vez & 15 & 15 \\
\hline \multirow{3}{*}{$\begin{array}{l}2 \text { ¿Crees que las dinámicas y juegos } \\
\text { realizados te ayudaron a aprender } \\
\text { cultura? }\end{array}$} & Sí & 87.50 & 75 \\
\hline & No & 7,50 & 25 \\
\hline & Tal vez & 5 & 0 \\
\hline \multirow{3}{*}{$\begin{array}{l}3 \text { ¿Aprendiste algo más sobre } \\
\text { cultura? }\end{array}$} & Nada & 2,50 & 0 \\
\hline & Poco & 47,50 & 50 \\
\hline & Mucho & 50 & 50 \\
\hline \multirow{5}{*}{$\begin{array}{l}4 \text { Escoge la forma con la que más te } \\
\text { interesa aprender sobre cultura }\end{array}$} & Memoria & 0 & 5 \\
\hline & Juegos & 50 & 70 \\
\hline & Videos & 42,5 & 25 \\
\hline & Libros/cuadernos & 7,50 & 0 \\
\hline & Otros & 0 & 0 \\
\hline \multirow{3}{*}{$\begin{array}{l}5 \text { ¿Pudiste conocer y aprender a } \\
\text { través del juego desarrollado } \\
\text { costumbres y tradiciones de un } \\
\text { pueblo? }\end{array}$} & Sí & 57,50 & 70 \\
\hline & No & 12,50 & 5 \\
\hline & Tal vez & 30 & 25 \\
\hline
\end{tabular}




\begin{tabular}{|c|c|c|c|}
\hline \multirow{3}{*}{$\begin{array}{l}6 \text { ¿Cómo te pareció el juego } \\
\text { realizado? }\end{array}$} & Educativo y divertido & 75 & 90 \\
\hline & Poco educativo y divertido & 15 & 10 \\
\hline & Nada educativo y divertido & 10 & 0 \\
\hline \multirow{4}{*}{$\begin{array}{l}7 \text { ¿Qué ventajas piensas que brindó el } \\
\text { juego al momento de realizarlo? } \\
\text { Escoge solo dos. }\end{array}$} & Inseguridad & 3,75 & 5 \\
\hline & $\begin{array}{rr}\text { Transmite costumbres } \quad y \\
\text { tradiciones }\end{array}$ & 47,50 & 45 \\
\hline & Es una pérdida de tiempo & 7,50 & 0 \\
\hline & $\begin{array}{l}\text { Desarrolla la comunicación } \\
\text { entre los compañeros/as y } \\
\text { docentes }\end{array}$ & 41,25 & 50 \\
\hline \multirow{5}{*}{$\begin{array}{l}8 \text { ¿Qué actividad escogerías para } \\
\text { aprender con mayor interés sobre la } \\
\text { cultura de un pueblo? Escoge solo una } \\
\text { opción }\end{array}$} & Danza & 7,50 & 30 \\
\hline & Dramatización & 25 & 20 \\
\hline & Juegos & 50 & 50 \\
\hline & Pintura & 15 & 0 \\
\hline & Otros & 2,5 & 0 \\
\hline \multirow{3}{*}{$\begin{array}{l}9 \text { ¿El juego te ayudó a conocer más a } \\
\text { tus compañeros y su realidad cultural? }\end{array}$} & Sí & 50 & 50 \\
\hline & No & 27,5 & 10 \\
\hline & Tal vez & 22,5 & 40 \\
\hline \multirow{3}{*}{$\begin{array}{l}10 \text { ¿Te gustaría conocer más juegos } \\
\text { que te ayuden a entender la cultura de } \\
\text { tu país y del mundo? }\end{array}$} & Sí & 85 & 80 \\
\hline & No & 5 & 5 \\
\hline & Tal vez & 10 & 15 \\
\hline
\end{tabular}

En los resultados se puede evidenciar la prevalencia del juego en el aprendizaje cultural.

Fuente: Elaboración propia.

Bajo esta tesitura, al preguntar a los alumnos cuál es el medio que les parece más interesante para aprender cultura, más del $50 \%$ seleccionó al juego, con un porcentaje del $50 \%$ en ambos casos. El 50\% de los encuestados en ambos subniveles respondieron que el juego les ayudó a conocer más a sus compañeros y su realidad cultural, razón por la cual los porcentajes de la última pregunta del postest tuvieron mucho valor, ya que el $85 \%$ de niños y el $80 \%$ den adolescentes expresaron que si les gustaría aprender más juegos y dinámicas.

\section{CONCLUSIONES}

La cultura y el juego son referentes para el mejoramiento de la enseñanza-aprendizaje a nivel educativo, estos términos están relacionados históricamente, por un lado, los seres humanos a través de los tiempos han transmitido sus costumbres y tradiciones mediante actividades individuales y sociales; y por otro, los centros educativos han sido parte de esta transmisión cultural, convirtiéndose en lugares en donde se puede forjar la cultura ancestral. 
En suma, se puede decir que el uso del juego cultural en las aulas de clase permitió catalizar el aprendizaje cultural, tal es el caso que de acuerdo a datos obtenidos del pretest y del postest, se evidencia el cumplimiento del primer objetivo específico ya que antes de aplicar las metodologías lúdicas en el aula, el conocimiento cultural era mínimo, además, los estudiantes no tenían mayor interés por ampliar su campo cognoscitivo; por el contrario, luego de la aplicación del juego se pudo conocer, mediante el postest, que la experiencia lúdica fuese un medio clave para que el conocimiento y el aumento del interés por la cultura.

Respecto al segundo objetivo específico, se puede admitir que el sistema educativo en temas culturales todavía mantiene aspectos tradicionales de la vieja escuela. Tanto niños y adolescentes coinciden en la necesidad de aprender cultura en un espacio de educación formal, en donde se manejen metodologías activas que les permitan formar parte de su aprendizaje. Luego se pudo notar que el aprendizaje de los alumnos en cultura fue mucho más interesante que otras actividades como la danza, pintura, dramatización. Tanto niños como adolescentes coincidieron en que la actividad lúdica debe incluirse en la enseñanza cultural.

La principal novedad del estudio es que independientemente de la procedencia del juego, la inclusión de dinámicas lúdicas en el proceso de aprendizaje de la cultura debe ser una premisa sine qua non asimilada por las instituciones tanto públicas como privadas. De igual modo, y aunque existan otro tipo de actividades que pudieran albergar la enseñanza cultural, los juegos se ensalzan como una opción didáctica cercana y efectiva para el aumento del interés sobre el ámbito de la cultura.

Entre las limitaciones evidenciadas durante la realización del estudio estuvo el hecho de conocer las regulaciones y obtener las autorizaciones respectivas por parte de las autoridades de las instituciones, especialmente en la institución pública para proceder con esta actividad "extracurricular", asimismo, otras de las contingencias que dificultaron el desarrollo de las actividades lúdicas fueron la búsqueda de una muestra significativa y la aplicación del postest, esta última fue un obstáculo debido a que los estudiantes comenzaron a dispersarse luego que la actividad culminó. Se recomienda a futuros investigadores que se indiquen con claridad las normas del juego, especialmente aclarar el momento de reflexión posterior a la finalización de la actividad y por supuesto, de igual manera buscar la correlación con otras variables como la motivación y a su vez extrapolar el análisis a estudiantes de niveles inferiores como educación preparatoria y educación elemental.

\section{REFERENCIAS BIBLIOGRÁFICAS}

Aizencang, N. (2005). Jugar, aprender y enseñar: relaciones que potencian los aprendizajes escolares. Ediciones Manantial.

Bantulá, J. \& Mora, J. M. (2016). Juegos multiculturales: 225 juegos tradicionales para un mundo global (2a. ed.). Editorial Paidotribo México.

Bermúdez, D., Díaz, D. \& Fernández Bermúdez, D. (2018). El arte, el deporte y la lúdica: elementos claves de la educación para la paz. Revista Conrado, 14(63), 171-176. Recuperado a partir de https://conrado.ucf.edu.cu/index.php/conrado/article/view/736

Borroto, L. (2017). Cultura y formación de la personalidad en la primera infancia. Estudios del Desarrollo Social: Cuba y América Latina, 5(2), 34-39.

Bruner, J. (Ed.). (2015). La educación, puerta de la cultura. Editorial Antonio Machado Libros. 
Cañizares, J. M. \& Carbonero, C. (2016). Juegos y deportes populares, autóctonos y tradicionales. Editorial Wanceulen.

Capretti, S. (2011). La cultura en juego: El deporte en la sociedad moderna y post] moderna. Trabajo y sociedad: Indagaciones sobre el empleo, la cultura y las prácticas políticas en sociedades segmentadas, (16), 14-20.

Castorina, J. A. \& Dubrovsky, S. (2004). Psicología, cultura y educación: perspectivas desde la obra de Vigotsky. Editorial Noveduc.

Ferro, J. (2013). Educación y cultura. Editorial Universidad del Norte.

Hernández Sampieri, R., Fernández, C. \& Baptista, P. (2010). Metodología de la investigación. McGraw-Hill.

Ley Orgánica de Educación Intercultural. Dirección Nacional de Normativa Jurídico Educativa, Quito, Ecuador, 31 de marzo del 2011. Recuperado de https://educacion.gob.ec/documentoslegales-y-normativos/

Loos, S. \& Metref, K. (2007). Jugando se aprende mucho: Expresar y descubrir a través del juego. Editorial Narcea.

Marcaida, P., Carreño, M. \& Bermúdez, M. (2013). El juego como necesidad de la sociedad infantil. Revista Conrado, 9(39).

Marín, I. (2018). ¿Jugamos?: Cómo el aprendizaje lúdico puede transformar la educación. Editorial Paidós Educación.

Martínez, B. (2008). El aprendizaje de la cultura y la cultura de aprender. Revista Convergencia, 15(48).

Martínez, N. (2015). Identidad cultural y educación. Revista Diálogos, 8(4), 65-87.

Megias, A. y Lozano, Ll. (2019). El juego infantil y su metodología. España: Editorial Editex.

Mestres, L. (2009). Educaweb. Madrid, España: Educaweb. Recuperado de https://www.educaweb. com/noticia/2009/11/30/diversidad-cultural-aulas-oportunidades-educativas-3986/

Minerva, C. (2002). El juego: una estrategia importante. Educere, 6(19), 289-291.

Olmos, H. (2002). Educar en Cultura. Revista Iberoamérica, Recuperado de https://www.oei.es/ historico/pensariberoamerica/ric00a06.htm

Papalia, D. \& Martorell, G. (2017). Desarrollo humano. Editorial McGraw-Hill Interamericana

Paredes, J. (2003). Juego, luego soy. Wanceulen.

Peñalva, S., Aguaded, I. \& Torres-Toukoumidis, Á. (2019). La gamificación en la universidad española. Una perspectiva educomunicativa. Revista Mediterránea de Comunicación/Mediterranean Journal of Communication, 10(1), 245-256.

Porro, J. (2014). Sociología del consumo cultural. Romero, R. (Ed.), Manual Atalaya de apoyo a la gestión cultural (pp. 2-10). Editorial ATALAYA.

Torres-Toukoumidis, Á., Ramírez-Montoya, M. S. \& Romero-Rodríguez, L. M. (2018). Valoración y evaluación de los Aprendizajes Basados en Juegos (GBL) en contextos e-learning. Education in the Knowledge Society, 19(4), 109-128.

Vial, J. (1988). Juego y educación. Las ludotecas. Editorial AKAL.

UNESCO. (2018). Indicadores de cultura para el desarrollo. Recuperado de https://en.unesco.org/ creativity/sites/creativity/files/cdis/resumen_analitico_ecuador_0_1.pdf. 